\title{
Quantum Field Theory Is Not Merely Quantum Mechanics Applied to Low Energy Effective Degrees of Freedom
}

\author{
Stefan Hollands*and Robert M. Wald ${ }^{\dagger}$ \\ Enrico Fermi Institute and Department of Physics \\ University of Chicago \\ 5640 S. Ellis Avenue, Chicago, IL 60637, USA
}

\begin{abstract}
It is commonly assumed that quantum field theory arises by applying ordinary quantum mechanics to the low energy effective degrees of freedom of a more fundamental theory defined at ultra-high-energy/short-wavelength scales. We shall argue here that, even for free quantum fields, there are holistic aspects of quantum field theory that cannot be properly understood in this manner. Specifically, the "subtractions" needed to define nonlinear polynomial functions of a free quantum field in curved spacetime are quite simple and natural from the quantum field theoretic point of view, but are at best extremely ad hoc and unnatural if viewed as independent renormalizations of individual modes of the field. We illustrate this point by contrasting the analysis of the Casimir effect, the renormalization of the stress-energy tensor in time-dependent spacetimes, and anomalies from the point of quantum field theory and from the point of view of quantum mechanics applied to the independent low energy modes of the field. Some implications for the cosmological constant problem are discussed.
\end{abstract}

Quantum field theory provides an excellent description of all phenomena observed in nature, at least down to the distance scales probed by present accelerators. Nevertheless, there is good reason to expect that it will break down at some distance scale $l_{0}$ (presumably of order of the Planck length) and be replaced by a more fundamental theory. The most compelling reason to expect such a breakdown comes from the quantization of gravity: Although a mathematically rigorous formulation of quantum field theory on a classical gravitational background can be given, it does not seem possible to formulate a quantum theory of the spacetime metric itself within the conventional framework of quantum field theory.

${ }^{*}$ stefan@gr.uchicago.edu

${ }^{\dagger}$ rmwa@midway.uchicago.edu 
Even if quantum field theory does not provide a fundamental description of nature, one can attempt to understand its success in describing low energy phenomena in much the same way as one can understand why the continuum theory of elasticity is successful in describing the long wavelength excitations of a crystal. In the case of a crystal, the continuum theory clearly breaks down at the scale of the lattice spacing. Nevertheless, starting from the fundamental lattice theory, one can derive an effective continuum theory that provides an accurate description of all aspects of the long wavelength degrees of freedom.

It is widely believed that quantum field theory similarly arises as an effective field theory from a more fundamental theory defined at ultra-high-energy/short-wavelength scales. We do not disagree with this viewpoint and, indeed, this viewpoint has led to many fruitful insights into the nature of quantum field theory. However, there is an associated view, which is implicit in many discussions, that quantum field theory is nothing more than quantum mechanics applied to the low energy effective degrees of freedom of the fundamental theory, in much the same way as elasticity theory is just classical (or quantum) mechanics applied to the effective degrees of freedom describing low energy excitations of a crystal. The main purpose of this paper is to point out that, even for a free quantum field, there are some fundamental features of quantum field theory that cannot be properly explained in this associated viewpoint.

These fundamental features concern the holistic nature of renormalization theory. For simplicity and definiteness, we focus our discussion on a free, massless, Klein-Gordon scalar quantum field

$$
\nabla^{a} \nabla_{a} \varphi=0
$$

in curved spacetime. (Similar arguments could be made for any other linear or nonlinear quantum field theory.) As is well known, infinities arise in the calculation of any field quantity $\Phi$ corresponding to a nonlinear polynomial expression in $\varphi$ and its derivatives. Therefore, "subtractions" must be done to give $\Phi$ a well defined, finite meaning. From the quantum field theoretic viewpoint, it is crucial that $\Phi$ be defined in a local and covariant manner 1], 2]. The requirement that the "subtraction" be done in a local and covariant manner greatly constrains the renormalization procedure. In particular, as proven in [1], it reduces the ambiguities in any polynomial quantity $\Phi$ to at most a finite number of parameters.

Let us now compare this situation with the picture obtained if we decompose the quantum field $\varphi$ into modes, and view these modes as independent degrees of freedom. First, we divide these modes into "(ultra-)high-energy/short-wavelength" modes $\left(\omega \gtrsim 1 / l_{0}\right)$ and "low energy modes" $\left(\omega \lesssim 1 / l_{0}\right)$, where $l_{0}$ denotes the physical length scale at which quantum field theory breaks down. It would be reasonable to assume $l_{0}$ to be of order the Planck scale, $l_{0} \sim\left(G \hbar / c^{3}\right)^{1 / 2}$, but we need not make any such assumption here. We do not know how to accurately describe the "ultra-high-energy/short-wavelength" modes using the presently known laws of physics, but we assume that these modes contribute negligibly to $\Phi$. On the other hand, we assume that the degrees of freedom corresponding to the low energy modes are described by ordinary quantum mechanics. As is well known, the sum of these low energy mode contributions to any given nonlinear polynomial $\Phi$ is absolutely enormous (see below). Thus, subtractions are needed to obtain reasonable renormalized field quan- 
tities. In this respect, the situation with regard to defining $\Phi$ by such a truncated mode sum calculation is neither better nor worse than the above field theoretic calculation; some renormalization is needed in both cases. However, there is an important difference here in how the renormalization is to be carried out. The decomposition of $\varphi$ into modes representing its individual degrees of freedom is necessarily global in character. From the point of view of an individual mode, there is therefore no natural way of enforcing the requirement that the subtractions be done in such a way that the resulting $\Phi$ is defined in a local and covariant manner. In other words, the local and covariant character of $\Phi$ is a property that depends on the sum of all of the renormalized modes. An individual mode will have no way of knowing whether its own subtraction is correct unless it "knows" how the subtractions are being done for all other modes. Of course, one can make use of the enormous available freedom to make arbitrary subtractions to cook up mode-by-mode renormalization schemes that, by construction, reproduce the field theoretic renormalization prescription. However, as we shall illustrate below, in curved spacetime, these schemes are necessarily so ad hoc and unnatural - far worse than the familiar "vacuum subtractions" of Minkowski spacetimethat it is very difficult to imagine that they could have any validity. The field theoretic renormalization prescription makes sense only from a holistic point of view, not from the point of view of individual modes. In this sense, even a free quantum field is much more than the sum of its dynamically independent parts, and quantum field theory is much more than merely quantum mechanics applied to the individual low energy degrees of freedom of the field.

The holistic nature of renormalization in quantum field theory is disguised in Minkowski spacetime on account of the fact that the locally and covariantly constructed Hadamard distribution $H\left(x, x^{\prime}\right)$ that enters the quantum field theoretic renormalization prescription [1] for $\Phi$ happens to equal the expection value of $\varphi(x) \varphi\left(x^{\prime}\right)$ in the Minkowski vacuum state $|0\rangle$. As a consequence, in Minkowski spacetime, the subtractions performed using $H\left(x, x^{\prime}\right)$ can be given a relatively simple mode-by-mode interpretation as "vacuum subtractions". However, no such accident occurs in a general curved spacetime. Indeed, in a general curved spacetime, $H\left(x, x^{\prime}\right)$ can be defined only locally (i.e., for $x^{\prime}$ close to $x$ ) and cannot be equal to the expection value of $\varphi(x) \varphi\left(x^{\prime}\right)$ in any state. Normal ordering with respect to some "vacuum state" cannot yield a correct renormalization prescription in a general curved spacetime - see remark (3) on P. 303 of [1] for a formal proof of this statement-and there is no reasonable mode-by-mode interpretation of the quantum field theoretic renormalization prescription.

Three concrete examples will serve to illustrate the above points. The first concerns the energy of a Klein-Gordon field, eq.(II), in a $1+1$ dimensional static spacetime $\left(M, g_{a b}\right)$ of spatial topology $S^{1}$, with metric of the form

$$
d s^{2}=-d t^{2}+L^{2} d \theta^{2}
$$

where the range of the $\theta$-coordinate is $[0,2 \pi)$. This metric describes a flat universe with closed spatial sections of size $2 \pi L$. As already mentioned above, the quantum field theoretic renormalization prescription for defining the stress-energy tensor, $T_{a b}$, of $\varphi$ involves a subtraction performed by using a locally and covariantly constructed Hadamard distribution 
$H\left(x, x^{\prime}\right)$; one subtracts from the "point-split" expression for $T_{a b}$ in terms of $\varphi(x) \varphi\left(x^{\prime}\right)$ a similar expression constructed using $H\left(x, x^{\prime}\right)$ and then takes the coincidence limit. However, since the spacetime metric (2) is locally flat, for $x^{\prime}$ near $x$ the locally constructed Hadamard distribution $H\left(x, x^{\prime}\right)$ for the spacetime $\left(M, g_{a b}\right)$ must be identical to the corresponding locally constructed Hadamard distribution for two-dimensional Minkowski spacetime. This means that the renormalization prescription for $T_{a b}$ can be given the interpretation of a "vacuum subtraction", but what is being subtracted is not the vacuum energy of the modes of $\varphi$ that are actually present in $\left(M, g_{a b}\right)$ but rather the vacuum energy of the modes that hypothetically would have been present if the spacetime were globally Minkowskian [3]! From the quantum field theoretic point of view, this prescription is entirely natural, since the construction of the stress-energy tensor should be local in the spacetime metric $g_{a b}$ and the quantum field $\varphi$, and it therefore should not care about the global topology of $M$. But, in terms of the individual globally defined modes, this prescription makes no sense: Why should the appropriate subtractions be based upon the energies of modes in some fictitious Minkowski spacetime rather than the energies of the modes that are present in the actual universe $\left(M, g_{a b}\right)$ ?

Using the quantum field theoretic renormalization methods, one finds that the total energy, $E_{C}$, of the ground state ${ }^{1}$ is given by [4]

$$
E_{C}=-\frac{1}{12 L}
$$

This result is in close analogy with the Casimir effect for a field confined by conducting plates, which has been verified experimentally [5]. The negative value of $E_{C}$ can be understood to arise from the fact that there are, in a sense, fewer low energy modes of $\varphi$ in the universe $\left(M, g_{a b}\right)$ than in Minkowski spacetime, so the Minkowski subtraction overcompensates for the vacuum energy of the modes in $\left(M, g_{a b}\right)$.

Let us now attempt to reproduce eq.(3) energy by applying quantum mechanics to the low energy degrees of freedom of the quantum field $\varphi$. If one decomposes $\varphi$ into its spatial Fourier modes, one finds that the $n$th Fourier mode is precisely a harmonic oscillator with frequency

$$
\omega_{n}=\frac{n}{L} .
$$

Therefore, if each mode is put in its ground state but we only count modes with frequency $\omega \lesssim 1 / l_{0}$, we obtain the total energy

$$
E_{0}=\sum_{n=0}^{L / l_{0}} \frac{1}{2} \omega_{n}=\sum_{n=0}^{L / l_{0}} \frac{n}{2 L} \approx \frac{1}{4 L}\left(L / l_{0}\right)^{2} .
$$

This disagrees with eq. (3) not only in sign, but also by a factor of $\left(L / l_{0}\right)^{2} \gg 1$.

\footnotetext{
${ }^{1}$ The massless Klein-Gordon field does not actually have a ground state on account of the presence of a spatally homogeneous mode which grows linearly with time. This "infrared divergence" is not relevant to any of our considerations. All of our results rigorously apply to the Klein-Gordon field of mass $m$ (which does have a ground state) in the limit as $m \rightarrow 0$.
} 
The above colossal value obtained for $E_{0}$ by this calculation is, of course, very well known and comprises what is usually referred to as the "cosmological constant ${ }^{2}$ problem". However, we can attempt to "fix" the discrepancy between eqs. (3) and (51) by adjusting the zero of energy of the $n$th mode of the quantum field by an amount $\epsilon_{0}(n, L)$. As previously mentioned, the fact that one has to do a subtraction here is not, by itself, necesarily worse than what was done in the quantum field theoretic calculation, where a subtraction also was necessary. The revised formula for the total energy of the ground state would then be

$$
E_{0}^{\prime}=\sum_{n=0}^{L / l_{0}}\left[\frac{n}{2 L}-\epsilon_{0}(n, L)\right] .
$$

Clearly, with unconstrained freedom on the choice of $\epsilon_{0}(n, L)$, there is no difficulty in arranging for any answer that one wishes to get for $E_{0}^{\prime}$. Thus, one can, of course, make choices of $\epsilon_{0}(n, L)$ that yield agreement between the right sides of eqs. (3) and (6). The difficulty is that there are infinitely many ways of doing this, and none of them appear to be in any way natural. Indeed, the following would appear to be two very natural conditions to impose on $\epsilon_{0}(n, L)$. First, on a account of the invariance of $\varphi$ under a scaling of the spacetime metric (2), it would be natural to require $\epsilon_{0}(n, L)$ to respect this scaling and therefore be of the form $\epsilon_{0}(n, L)=f(n) / L$. Second, since the mode labeled by the integer $N n$ in a universe of size $2 \pi N L$ is locally identical (up to normalization) to the mode labeled by integer $n$ in a universe of size $2 \pi L$, it would be natural to require $\epsilon_{0}(n, L)$ to respect this fact by depending on $n$ and $L$ only in the form $n / L$. However, these two requirements would constrain $\epsilon_{0}(n, L)$ to be of the form $c n / L$ for some constant $c$. But this choice would then yield $E_{0}^{\prime} \approx(1-2 c) L / 4 l_{0}^{2}$, which does not agree with eq. (3) for any choice of $c$. Thus, these "natural" requirements on $\epsilon_{0}(n, L)$ are incompatible with the Casimir effect. We do not believe that the Casimir effect can be understood without invoking the holistic aspects of quantum field theory.

Our second example concerns the general problem of defining the renormalized stressenergy tensor, $T_{a b}$, in a general, time-dependent, globally hyperbolic, curved spacetime. We have just argued that even in a static flat spacetime, the construction of $T_{a b}$ cannot be understood without invoking the holistic aspects of quantum field theory, so the situation in a general curved spacetime cannot be better. However, we wish to point out that the general situation is actually far worse, i.e., one must go to much greater lengths to attempt to account for the stress-energy of quantum field by subtractions performed on its individual degrees of freedom.

From the quantum field theoretic point of view, the calculation of $T_{a b}$ in a general curved spacetime proceeds in much the same way as indicated above for the Casimir effect. One

\footnotetext{
${ }^{2}$ The reason for this terminology is that, in Minkowski spacetime, by Lorentz invariance, the expected stress-energy in the vacuum state must be proportional to the metric. Therefore, an absurdly large value of $<T_{a b}>$ would correspond to the presence of an absurdly large value of the cosmological constant in Einstein's equation. In fact, the insertion of a high energy cutoff as we have done here breaks Lorentz invariance and - since each individual mode contributes a traceless stress-energy tensor - it is easy to see that the unrenormalized mode sum for $T_{a b}$ corresponding to eq.(15) would not be proportional to $g_{a b}$. Nevertheless, we will use the conventional terminology in referring to the enormous value of $E_{0}$ as the "cosmological constant problem".
} 
again performs a suitable subtraction using a locally and covariantly constructed Hadamard distribution $H\left(x, x^{\prime}\right)$. The only additional complication is that when spacetime curvature is present, there is now a small amount of additional freedom in the renormalization prescription, which allows one to modify the final result for $T_{a b}$ by the addition of conserved local curvature tensors of the correct scaling dimension [6]. In 4-dimensions, there are two such curvature tensors, so there is a two-parameter freedom ${ }^{3}$ in the definition of $T_{a b}$.

Let us now try to construct $T_{a b}$ by applying quantum mechanics to the low energy modes of $\varphi$, without invoking any holistic aspects of quantum field theory. We immediately face a serious problem in that it is far from clear how to even define the "modes" of $\varphi$ : The decomposition of a quantum field into modes requires a definition of "positive frequency", but there is no natural positive/negative frequency decomposition of $\varphi$ in a non-stationary spacetime. Nevertheless, we can proceed by making some arbitrary choice of "positive frequency", corresponding to some arbitrary choice of "vacuum state" $|0\rangle$. We can then write down a mode sum formula for $\left\langle 0\left|T_{a b}\right| 0\right\rangle$ analogous to eq. (15). As in eq. (15), we will obtain an enormous value for $\left\langle 0\left|T_{a b}\right| 0\right\rangle$, so some subtractions are needed. But it is hard to imagine that there could be any natural rule on what to subtract from each mode that would yield agreement with the quantum field theoretic expression. In particular, as already noted above, subtraction of the entire vacuum stress-energy - i.e., normal ordering - is incompatible with the quantum field theoretic prescription. Furthermore, taking into account the known dependence of $H\left(x, x^{\prime}\right)$ on the spacetime metric, it is not difficult to see that in $D$ dimensions, the quantum field theoretic prescription for defining $T_{a b}$ involves the local subtraction of terms that depend upon the derivatives of the metric up to $D$ th order. This means that if we consider a two-dimensional spacetime with metric of the form (2) but with $L$ now allowed to depend upon $t$, then in order to reproduce the quantum field theoretic prediction for energy, it would be necessary for the "vacuum energy subtraction" $\epsilon_{0}$ to depend not only on $n$ and $L$ but also on $d L / d t$ and $d^{2} L / d t^{2}$ - even though the Hamiltonian for the individual modes depends only on $n$ and $L$. Again, we do not believe that it is possible to sensibly derive the quantum field theoretic prediction for $T_{a b}$ without invoking the holistic nature of quantum field theory.

Our final example concerns anomalies. One of the most surprising aspects of quantum field theory is that certain relations involving the field equations that are manifestly true in classical field theory cannot be satisfied in quantum field theory. For example, in the case of a Klein-Gordon field $\varphi$ in 4-dimensional curved spacetimes, although eq. (11) of course holds, it is impossible to define the renormalizations so as to satisfy both of the following relations [7]

$$
\begin{gathered}
\varphi \nabla^{a} \nabla_{a} \varphi=0 \\
\nabla_{b} \varphi \nabla^{a} \nabla_{a} \varphi=0 .
\end{gathered}
$$

(In the case of a conformally invariant field, the similar inability to simultaneously impose

\footnotetext{
${ }^{3}$ If we were to consider a massive Klein-Gordon field, then we also would have the additional freedom to modify the definition of $T_{a b}$ by terms of the form $m^{4} g_{a b}$ and $m^{2} G_{a b}$.
} 
analogs of eqs. (17) and (8) is responsible for the existence of a trace anomaly in the stressenergy tensor of that field.) From the quantum field theoretic point of view, the above anomaly arises because in a general curved spacetime it is impossible to locally construct a Hadamard distribution $H\left(x, x^{\prime}\right)$ that satisfies the Klein-Gordon equation in both $x$ and $x^{\prime}$ 8]. Consequently, the quantum field theoretic subtraction procedure fails to fully respect the Klein-Gordon equation.

Any attempt to reproduce the above anomaly by applying quantum mechanics to the low energy modes of $\varphi$ would have to be truly bizarre. Each term in the mode sum formulas for the left sides of eqs. (77) and (8) would vanish, since the individual modes themselves do not suffer any anomalies. Yet, one would nevertheless have to do some "subtraction" to obtain agreement with the quantum field theoretic prediction. Again, we do not believe that the existence of anomalies can be understood without invoking the holistic nature of quantum field theory.

As already mentioned above, the absurdly large value obtained for the stress-energy of a quantum field when computed by applying quantum mechanics without subtractions to the low energy modes of the field is usually referred to as the "cosmological constant problem". In 4 dimensions, a calculation similar to that leading to eq. (5) above would yield an expected energy density of order $1 / l_{0}^{4}$, where $1 / l_{0} \sim 10^{19} \mathrm{GeV}$ if $l_{0}$ is assumed to be of order the Planck length. By contrast, the actual energy density of our universe is only of order $\left[\sim 10^{-12} \mathrm{GeV}\right]^{4}$. If one were to view a quantum field as a collection of independent degrees of freedom that know nothing about each other, then it is hard to imagine how - even allowing for reasonable renormalization "subtractions" of the individual modes - cancellations of this magnitude could occur. Furthermore, in the absence of some exact, unbroken symmetry such as supersymmetry, it is equally hard to imagine how cancellations of this magnitude could occur between different fields. Thus, the enormous discrepancy between the naive mode-sum calculation and the observed energy density is therefore generally viewed as a very serious "problem". We do not share this view. As we have argued above, there are many aspects of the theory of a quantum field that simply cannot be understood by viewing its low energy degrees of freedom as being independent. The mode sum calculations like the one leading to eq. (5) do not properly take into account the holistic aspects of quantum field theory. In our view, it would be more fruitful to simply accept the holistic aspects of quantum field theory rather than search for models where-by virtue of miraculous cancellations - the holistic aspects do not need to be invoked ${ }^{4}$.

If one accepts the holistic aspects of quantum field theory, there is still a "cosmological constant problem", but it is rather different than the usual formulation of it. The puzzle is not, "Why is the observed energy density of the universe so small?" This is only a puzzle if one believes that it should be correct to calculate the stress-energy of a quantum field by treating its low energy modes as completely independent degrees of freedom that know nothing about each other, in which case implausible cancellations would be required.

\footnotetext{
${ }^{4}$ Of course, it remains a very significant puzzle as to why quantum field theory possesses holistic aspects, i.e., how they arise from the more fundamental, underlying theory. However, it is likely that we will need a much deeper understanding of the underlying theory in order to account for this.
} 
Rather, the puzzle is, "Why is the cosmological constant so large?" Quantum field theory predicts that the stress-energy tensor of a free quantum field in an adiabatic vacuum state in a slowly expanding 4-dimensional universe should be of order of $L^{-4}$, where $L$ denotes the size and/or radius of curvature of the universe. For our universe, $1 / L$ would be of order $\sim 10^{-42} \mathrm{GeV}$. But observations of type Ia supernovae and the cosmic microwave background strongly suggest that, at the present time, the dominant component of stress-energy in the universe is smoothly distributed (i.e., not clustered with galaxies) and has negative pressure. The energy density of this so-called "dark energy" is thus $\left[\sim 10^{-12} \mathrm{GeV}\right]^{4}$, i.e. roughly the geometric mean of the unsubtracted mode sum and quantum field theoretic predictions for vacuum energy density. It is, of course, not presently known whether this dark energy corresponds to the vacuum energy of some field, the potential energy of some field, some other form of matter, or simply corresponds to the presence of a cosmological constant term in Einstein's equation. In any case, however, it is seems very difficult to account for its energy scale. This is a true puzzle. We do not have any new proposals to make here concerning the nature of dark energy. However, if dark energy does correspond to vacuum energy of an interacting quantum field, it is our view that its properties will be understood only by fully taking into account the holistic nature of quantum field theory.

This research was supported in part by NSF grant PHY00-90138 to the University of Chicago.

\section{References}

[1] S. Hollands and R.M. Wald, Commun. Math. Phys. 223, 289-326 (2001); gr-qc/0103074.

[2] R. Brunetti, K. Fredenhagen, and R. Verch, Commun. Math. Phys. 237, 31-68 (2003); math-ph/0112041.

[3] B.S. Kay, Phys. Rev. D20, 3052-3062 (1979).

[4] N.D. Birrell and P.C.W. Davies, Quantum Fields in Curved Space, Cambridge University Press, (Cambridge, 1982).

[5] M. Bordag, U. Mohideen, and V.M. Mostepanenko, Phys. Rept. 353, 1-205 (2001); quant-ph/0106045.

[6] R.M. Wald Quantum Field Theory in Curved Spacetime and Black Hole Thermodynamics, University of Chicago Press, (Chicago, 1994).

[7] S. Hollands and R.M. Wald, "Conservation of the stress tensor in interacting quantum field theory in curved spacetime", arXiv:gr-qc/0404074.

[8] R.M. Wald, Phys. Rev. D17, 1477 (1978). 